\section{Boosting Newcastle disease vaccination efficacy under field conditions by aromatic plant essential oil extracts}

\author{
Mohammad S. Khalifeh, \\ Ehab A. Abu-Basha \\ Department of Basic Veterinary Medical \\ Science, Veterinary Science College, \\ Jordan University of Science and \\ Technology, Irbid, Jordan
}

\section{Abstract}

This study was performed to evaluate the effects of commercially available aromatic plant essential oil extracts (MixOil ${ }^{\mathrm{TM}}$ ) on the protection outcome achieved after a Newcastle disease (ND) vaccination. Antibody production, after a MixOil treatment administered along with a vaccination program applying a live attenuated Newcastle disease virus (NDV) vaccine, was assessed under field conditions. The antibody response was evaluated via a Hemagglutination Inhibition test and an Enzyme-Linked Immunosorbent Assay. Later, the experimental groups were challenged with two velogenic NDV strains: Herts 33 and a local virulent NDV strain. A MixOil treatment effect with a higher dose was also examined. It was observed that ND antibody titers were enhanced when the birds were placed on a MixOil immune system-boosting program during vaccination. The mortality rate decreased by at least $50 \%$ after regular MixOil immune boosting; it also decreased following larger treatment doses. Clearly, promoting a strong bird immune system through herbal supplementation would naturally be expected to create a successful vaccination outcome and healthy flocks of birds. In addition, the results suggest that, by applying a higher dose of MixOil, the treatment can exceed its immunestimulator benefits; it resulted in controlling the mortality outcome from the experimental ND infection.

\section{Introduction}

Essential oil therapeutic applications for various kinds of diseases have expanded worldwide. Their uses range from treating inflammatory diseases, such as allergies, rheumatism, and arthritis, to using them as antimicrobial agents. 1,2 These compounds do not carry nutritional values for animals, but they are essential to plants for defense against harsh environmental conditions. ${ }^{3,4}$
The application of herbal medicine in farm animals is limited; they are mostly considered to be food additives mainly used to concentrate on improving the production performance of livestock. However, many herbal medicine products claim to have beneficial effects on boosting or restoring animal health parameters in stressful and diseased conditions. Among these uses in the poultry industry, for example, is an investigation that employed a mannan-oligosaccharide, or an essential oil mixture, as a feed additive to the basal diet of laying hens. 5 Their results showed an improvement of egg quality and liberated the chickens from the negative effects of heat stress on weight gain. As an aside to improving production performance, the supplementation of these feed additives improves humoral immune response stimulation. ${ }^{5}$ An additional experiment showed that an herbal extract mixture of oregano, laurel leaf, and lavender oil can assist in lightening the deleterious effects that some parasites have on the production performance of broiler chicks. ${ }^{6}$ Several other experiments demonstrated that some herbal extract blends are to be valued in the reduction of fecal oocyst counts in birds infected with Eimeria tenella. ${ }^{6-8}$

The current study was conducted to evaluate the immune system-boosting effects of commercially available essential oil blends in response to ND vaccination and infection. This essential oil blend contains carvacrol, thymol, eucalyptol, and lemon, formulated in a homogenized mixture known as MixOil. ${ }^{9}$ Controlling the impact of Newcastle disease (ND) is a great challenge facing the poultry industry, especially in endemic areas. In general, vaccination and high biosecurity measures are meant to decrease substantial losses from high morbidity and mortality, especially those accompanied by various different diseases, including ND. However, in outbreaks with highly virulent NDV strains, losses are often tremendous and uncontrollable, even in vaccinated flocks. Many ND vaccination programs have been adopted in the field in these endemic areas, and farmers usually lose track of what the most beneficial programs are. The many intense programs applied in the field ultimately add extra stress to the members of each flock. Vaccination programs impact birds from birth, and many different combinations of vaccine strains, including NDV, are loaded into flocks. A chick's immune system is expected to deal with these wide ranges of infectious agents in the form of vaccinations and thus acquire protection against them. Consequently, a failure to maintain the healthy status of birds eventually leads to failure, even for the most successful vaccine and vaccination programs. Therefore, an introduction of organic compounds in the form of herbal supplements that help promote healthy bird
Correspondence: Mohammad S. Khalifeh, Department of Basic Veterinary Medical Science, Veterinary Science College, Jordan University of Science and Technology, P. 0. Box 3030, Irbid, 22110 Jordan.

Tel.: +962.2720.1000/22001; Fax: +962.2720.1081. E-mail: mskn@just.edu.jo

Key words: Newcastle disease vaccine, Newcastle disease treatment, antibody response and essential oil extracts.

Acknowledgments: this work was funded completely by Animal Wellness Products (AWP), Reggio Emilia - Italy. The authors acknowledge the technical help of Dr. Mohammad Khashan, the breeder unit veterinary supervisor at the AlJazeera Poultry Company, Amman - Jordan, as well as the supervisor of the JUST Animal House Unit, Mr. Mohammad Damra, and the animal caretaker team. In addition, the authors extend their appreciation to Mr. Ibrahim Al-Awadie, the owner of the Naoor Poultry Farm, where the field portion of this study was conducted.

Contributions: MSK designed the study, performed the statistical analysis, conducted the experiments and the laboratory work, and wrote the manuscript; EAAB assisted in experimental design, interpreting the results and writing the manuscript.

Conflict of interests: the authors declare no potential conflict of interests.

Received for publication: 12 Julu 2014.

Revision received: 26 August 2014.

Accepted for publication: 27 August 2014.

This work is licensed under a Creative Commons Attribution NonCommercial 3.0 License (CC BYNC 3.0).

(C) Copyright M.S. Khalifeh and E.A. Abu-Basha, 2014 Licensee PAGEPress srl, Italy

Veterinary Science Development 2014; 4:5546 doi:10.4081/vsd.2014.5546

immune systems should help lead to successful vaccination outcomes.

\section{Materials and Methods}

\section{The chickens and the vaccination program}

The testing of the Mix0il effect on immune response and NDV vaccination efficacy was performed in a field study at a semi-open rearing system in Jordan. The farm had two separate houses, each rearing 5800 Hubbard classic broilers. The farm used a live attenuated clone-30 NDV vaccine strain (Nobilis Ma5 \& 
Clone 30, Merck Animal Health, USA), which was administrated to the chickens via drinking water on days 1,14 , and 24 . The farm monitored for any health problems during the rearing period (40 days).

\section{The MixOil immune system-boost- ing program}

One of the rearing houses (Group 1), containing 5800 birds, received a MixOil treatment (Animal Wellness Products, Reggio Emilia, Italy). Another house (Group 2), with the same rearing conditions, health status and number of birds, served as a control group (i.e., no Mix0il treatment). The Group 1 birds received a Mix0il treatment in water, continuously for the first 10 days and for 3 days after each vaccine administration (e.g., day 14 to day 17 and day 24 to day 27). The recommended manufacture's dose for MixOil treatments is $0.25 \mathrm{~mL}$ per liter of water. The Mix0il concentrated liquid is formalized to disperse easily in water.

\section{The Newcastle disease virus chal- lenge studies}

The two challenge studies were executed in the Animal House Unit at the Jordan University of Science and Technology (JUST). One hundred and ten 26-day-old birds from each rearing house were transferred to the Animal House. The birds were kept in their experimental groups for four days to ensure their adaptation to their new environment before the beginning of the challenge experiment. They were kept in a controlled environment until the end of the first challenge study at day 45 of birds' age. The birds were either unexposed to a virus or intraocularly challenged with $10^{6.0} \mathrm{ELD}_{50}$ of the velogenic Herts 33 NDV strain on day 30 . The summary of the generated experimental groups and the number of replicates in each group is presented in Table 1 . The protection level is reflected by the resulting mortality rate after the challenge. Clinical signs and gross pathological lesions were recorded to confirm successful ND chal- lenges. All of the birds in the experimental groups that participated in the challenge experiments were maintained in strict isolation units and under similar management conditions, with feed and water ad libitum.

The second challenge study was performed on the control birds that were spared the Herts 33 challenge throughout the first challenge study. These birds were exposed to a local (Jordanian), highly virulent NDV isolate on day 45 at a dose of 106.5 EID50/bird. 10 The bird groups in this experimental extension are characterized in Table 1.

Chicks comparable to the birds present on the farms, in terms of breed and maternal antibody levels, were previously raised at the JUST Animal House; these birds were left without any NDV vaccination. At 30 days, these unvaccinated birds were also challenged, with either the Herts 33 neurotropic strain or the local Jordanian NDV strain, at a dose of $10^{6}$ EID50/bird. Although the controlled environment for these birds was not comparable to the ongoing field experiments, this portion of the work helped with recording the baseline mortality rate and the clinical signs that resulted from the NDV challenge in the controlled environment at the JUST Animal House for nonvaccinated birds.

\section{Sample collections}

Blood samples (2-3 mL) were collected into plain tubes from the left brachial vein. Serum samples were collected from each experimental farm unit on days $0,10,20,30$, and 40 (40 samples per group for each time point). Moreover, blood samples from those birds transferred to the JUST Animal House for the purpose of the experimental challenges were collected from the infected and non-infected birds on days 30 and 45 .

\section{The MixOil treatment program}

Some birds in the NDV challenge studies received four times the recommended MixOil dose, orally in water, on the same challenge day until the end of the protection assessment period (i.e., 15 days after the challenge) (Table 1). The purpose of increasing the MixOil dose at the time of the challenge was to highlight any potential benefits that were expected to arise from this product in terms of controlling the deleterious effects of NDV infection. The dose implemented was within the safety margin dose approved by the manufacturer. The Mix0il treatment, at normal manufacture and at four times the MixOil recommended dose, were labeled $1 X$ and $4 X$, respectively.

\section{The immune response evaluation}

The serum samples were evaluated for the presence of any antibody response, specifically against NDV, using the Hemagglutination Inhibition (HI) method and Enzyme-Linked Immunosorbent Assay (ELISA). The reciprocal of the last serum dilution, showing an inhibition of hemagglutination, was considered the $\mathrm{HI}$ antibody titer of the serum. Indirect ELISA (ProFLOK NDV Plus, Location), specific for the IgG anti-NDV isotype, was performed according to the manufacturer's recommendation (Synbiotics, USA). The antibody titer was calculated through company software, which correlates the optical density of the samples with the positive control optical density (i.e., the sample/positive ratio).

\section{Statistical analysis}

Results were compared using a two-way analysis of variance, and significant differences among means were tested using a student's $t$-test. For all tests and comparisons, the difference was considered significant at a value of $\mathrm{P}<0.05$.

\section{Results}

\section{Maternal antibody profile under field conditions}

The maternal antibody titer in the experimental groups raised at the farm, as detected by ELISA on day 1 , was $9385.55 \pm 223.64$, while

Table 1. Experimental design for the challenge study with velogenic Herts 33 or a local velogenic Newcastle disease virus strain.

\begin{tabular}{|c|c|c|c|c|}
\hline \multirow{2}{*}{ Before challenge } & \multirow[b]{2}{*}{ After challenge } & \multicolumn{2}{|c|}{$\begin{array}{c}\text { Herts } 33 \text { challenge } \\
\text { experiment } \\
\text { (number of birds)* }\end{array}$} & \multirow{2}{*}{$\begin{array}{l}\text { Local strain challenge } \\
\text { experiment } \\
\text { (number of birds) }^{\circ} \\
\text { Challenged }\end{array}$} \\
\hline & & Challenged & Not challenged & \\
\hline None & None & 35 & $20^{\circ}$ & 10 \\
\hline $1 X^{\#}$ & None & 35 & $20^{\circ}$ & 10 \\
\hline None & $4 X^{\S}$ & 35 & 20 & 10 \\
\hline $1 \mathrm{X}$ & $4 X$ & 35 & 20 & 10 \\
\hline
\end{tabular}


the anti-log HI unit was $200 \pm 13.1$. In the nonvaccinated birds, the antibody level on day 1 was not significantly different from that of the birds that were vaccinated on day 1 and nurtured at the farm, even though they were raised in a different time frame (i.e., two months earlier than the field study). The antibody level, detected at an early age after vaccination, showed that the antibody detection by the HI test disappeared by day 10 (Figure 1A). However, the detection of antibodies by ELISA on day 10 showed some antibody titer in both field experimental groups (Figure 1B). A similar trend was observed in the non-vaccinated group at the same time point (10 days of age), but the antibody response completely disappeared at the later-tested time points for these non-vaccinated birds (data not shown).

\section{The humoral immune responses elicited by Newcastle disease vac- cines}

Antibody titers were considered positive if the anti-log2 HI unit was higher than 64 , which equals $6 \mathrm{HI}$ units, while an antibody titer of 1800 and above as detected by ELISA was considered protective, according to the manufacture's interpretation. After three field vaccination injections of a live attenuated Clone-30 NDV, only a slight peak of antibody production was noticed at 30 days of age. The peak showed an anti-log2 HI unit below 64, while the antibody titer detected by ELISA reached 1100 (Figure 1).

\section{The effect of MixOil on the humoral responses elicited by Newcastle disease vaccines}

The antibody assessment after the MixOil introduction at the manufacture's recommended dose (i.e., 1X) to the vaccinated flocks showed a significant increase of specific antibody responses against NDV above that of the untreated group. It is obvious that the $1 \mathrm{X}$ MixOil treatment had a prominent antibody production, greater than that of the untreated group, at 30,40 , and 45 days of age (Figures $1 \mathrm{~A}$ and $2 \mathrm{~A}$ ). In addition, the treatment with $1 \mathrm{X}$ MixOil, along with vaccination, resulted in an anti-log2 $\mathrm{HI}$ unit that was slightly below 64 at 30 and 40 days of age (Figure 1A). The antibody titer detected by ELISA was higher in the Mix0il-treated group from day 20 until the end of the experiment (Figure 1B). The ELISA antibody titer in the MixOil-treated group crossed the manufacturer-determined protective titer threshold on day 30. The untreated MixOil group had only a slight non-protective peak of antibody production at 30 days of age. The treatment in the Mix0il group resulted in sustained antibody production from day 30 until the end of the experiment (Figures 1B and 2B).
The effects of vaccination on protection against an Newcastle disease virus experimental challenge

Previous challenge experiments with the Herts 33 neurotropic strain and the local Jordanian NDV strain in the controlled environment at the JUST Animal House led to a high mortality rate in the unvaccinated birds. The mortality rate after the challenge with these viruses in the JUST Animal House environment usually started to appear five days after the challenge and reached its maximum three to four days later. The mortality rate in the non-vaccinated birds after exposure to the Herts 33 neurotropic strain and the local Jordanian NDV strain, which was around 90\%, was reached during the protection assessment period (i.e., 15 days). The applied vaccination program had a mortality rate following exposure to the Herts 33 neurotropic strain challenge around $28 \%$ while the challenge with the local Jordanian NDV strain resulted in mortality rate as high as $80 \%$ (Table 2 ).

\section{MixOil protective treatment effects against the Newcastle disease virus experimental challenge}

The mortality rate after the experimental NDV infection was limited, and decreased by $50 \%$ in the group that was treated with the MixOil immune system-boosting program at the farm and had no further exposure to the Mix0il treatment after the challenge (Table 2). A clear benefit of the MixOil treatment was

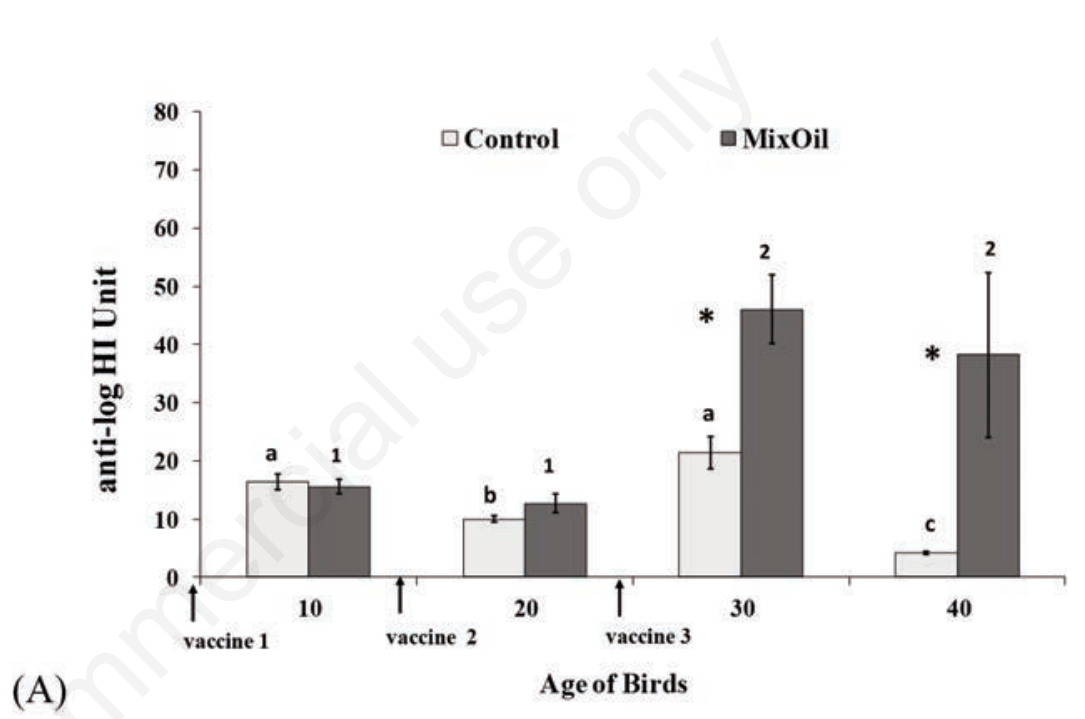

(A)

Age of Birds

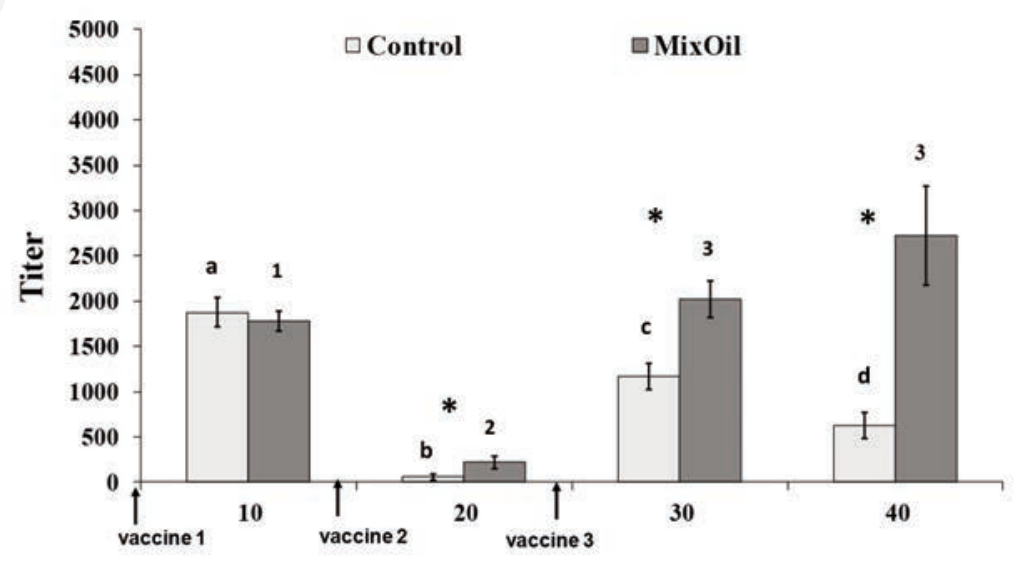

(B)

Age of birds

Figure 1. Newcastle antibody titers in serum after a MixOil treatment under field conditions. The birds were administered three live attenuated NDV vaccinations in drinking water on days 1, 14, and 24. The antibody titer was represented by an antilogarithmic scale of the HI unit (A) and by ELISA (B). The values are mean \pm S.E. Statistical comparison across time is represented by letters for the control group and by numbers for the MixOil group. The asterisks represent significant differences between the two experimental groups within each time point, while different letters or numbers indicate a significant difference for each group across time with a $P$ value less than 0.05 . 
obvious when the birds received a $4 \mathrm{X}$ dose at the time of the challenge with the Herts 33 neurotropic strain (Table 2). Therefore, a combination of the MixOil immune system-boosting program (i.e., $1 \mathrm{X}$ ) that was applied at the farm and the 4X MixOil dose at the time of the challenge resulted in a $70 \%$ decrease in the mortality rate (e.g., mortality rate $=8.6$ ), when compared to that of the MixOil-untreated but NDV-vaccinated group.

It was clear that a high mortality rate was achieved after the challenge with the local Jordanian NDV strain. The MixOil treatment benefits, after the challenge with the local Jordanian NDV isolate, followed a similar trend as the challenge with the Herts 33 neurotropic strain (Table 2). The accomplished decrease in the mortality rate was best when the $4 \mathrm{X}$ MixOil treatment dose was applied.

\section{The effect of MixOil on the} humoral responses elicited by the Newcastle disease virus challenge

The NDV experimental infection resulted in a significant up-regulation in the antibody production in all groups (Figure 2). The infected groups that previously received the MixOil treatment at the farm had a lower antibody production than those of the MixOil-untreated but infected groups, regardless of the later application of the $4 \mathrm{X}$ dose at the time of infection. Therefore, the birds that were infected with the virulent NDV strain and received the 4X Mix0il treatment at the time of the challenge had a lower antibody production detected by the HAI test than that of the infected MixOil-untreated control birds (Figure 2A). Still, this group had a higher antibody production than the groups treated with 1X MixOil and at the farm (Groups 1X or $1 \mathrm{X}+4 \mathrm{X}$ ). An ELISA analysis of antibody production revealed that the Mix0il treatment used as an immune booster course (1X) at the farm, in addition to the vaccination, had lower antibody production than that of the MixOil-untreated groups after experimental challenge (Figure 2B). The behavior of the antibody profile as detected by ELISA was similar to that of the antibody production detected by HAI.

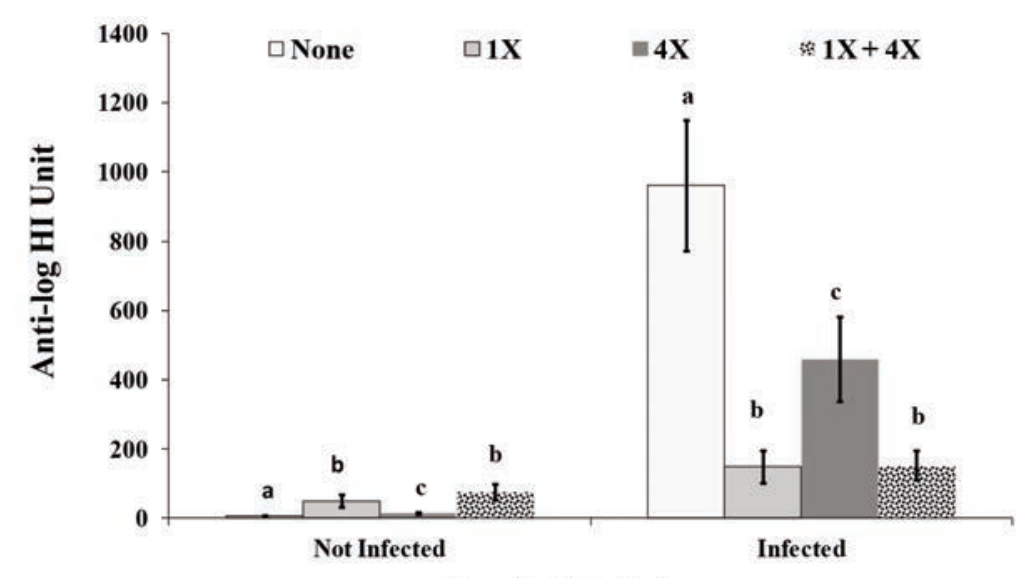

(A)

Day 45 of Birds Age

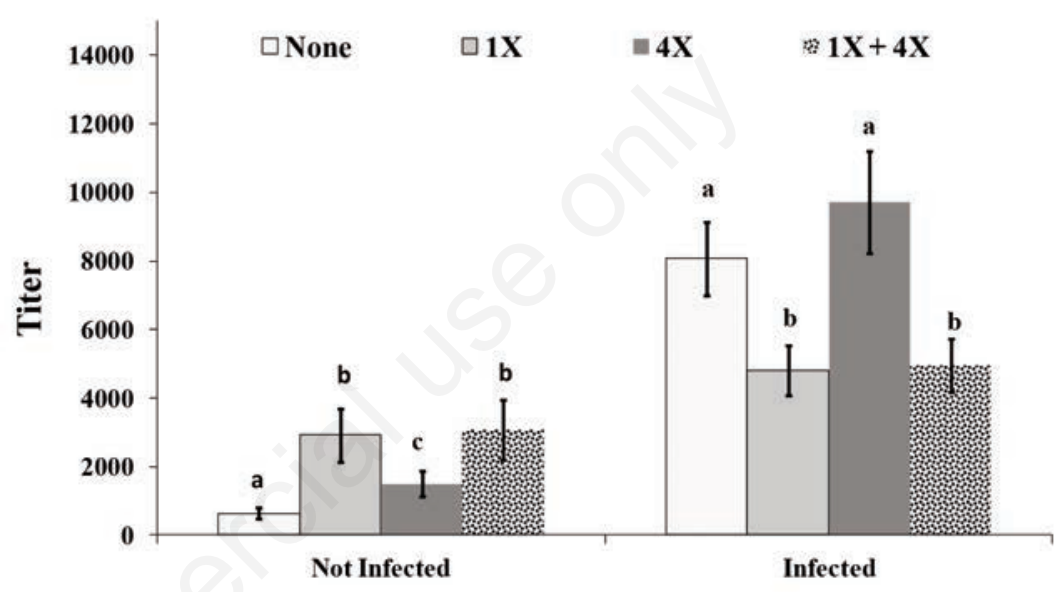

(B)

Day 45 of Birds Age

Figure 2. A comparison of the immune response generated after infection with a velogenic NDV strain. The antibody response was measured by an $\mathrm{HI}$ test; the $\mathrm{HI}$ unit is represented by the antilogarithmic scale (A) and by ELISA (B). All birds at the farm received vaccinations in drinking water with three live attenuated NDV vaccinations on days 1, 14, and 24. Vaccinated birds were either infected on day 30 or left uninfected at the JUST AH facilities. A comparison was made between the experimental groups that received various MixOil treatments at the farm in the immune system-boosting program (1X) or when treated with the $4 \mathrm{X}$ dose after experimental infection. Mean values with different letters are statistically different $(\mathbf{P}<0.05)$ from other treatment groups within the same experimental group (values with the same letters were not significantly different).

Table 2. Newcastle disease virus vaccination protection efficacy after the application of the MixOil immune system-boosting and treatment programs.

\begin{tabular}{|c|c|c|c|c|c|}
\hline \multicolumn{2}{|c|}{ MixOil dose (time of application)* } & \multicolumn{2}{|c|}{ Herts 33 challenge ${ }^{\circ}$} & \multicolumn{2}{|c|}{ Local strain challenge ${ }^{\circ}$} \\
\hline Before challenge & After challenge & Dead/Total & $\%$ & Dead/total & $\%$ \\
\hline None & None & $10 / 35$ & 28.6 & $08 / 10$ & 80 \\
\hline $1 X^{\#}$ & None & $5 / 35$ & 14.3 & $06 / 10$ & 60 \\
\hline None & $4 X \S$ & $4 / 35$ & 11.4 & $03 / 10$ & 30 \\
\hline 1X & $4 \mathrm{X}$ & $3 / 35$ & 8.6 & $03 / 10$ & 30 \\
\hline
\end{tabular}

*All MixOil-treated birds received in drinking water three oral vaccinations with a live attenuated Clone- 30 on days 1,14 , and 24 . $^{\circ}$ Oculonasal challenge at 30 days old with a Herts 33 NDV strain and with a local virulent NDV strain at 45 days old for the control non infected birds spared from the first Herts 33 NDV strain challenge study. *Birds received the manufacturer-recommended dose of MixOil in the immune system-boosting program applied in the field before the experimental challenge. sBirds received MixOil at four times the manufacturer-recommended dose in the treatment program applied after experimental challenge. 


\section{Discussion}

The results presented in the current study showed that aromatic plant essential oil extract (MixOil) supplementation in the drinking water administrated to chickens can boost the humoral immune stimulation response after NDV vaccinations. Moreover, it had a clear benefit in raising the protection outcome of the vaccination after the experimental infection. Regardless of the detection methods applied, an up-regulation in antibody titer was achieved after oral MixOil treatment. This claim is demonstrated and reinforced in similar studies that used herbal extracts to boost immunity. Among these studies, some even promote the use of herbal extract supplementation as an immune adjuvant.11,12 Additionally, it has also been suggested that the successful replacement of ordinary adjuvants with an herbal extract supplement is expected to overcome some of the side effects and shortcomings of various ordinary adjuvants in stimulating the proper immune effects against some antigens. ${ }^{11,12}$

Chinese herbal ingredients, subcutaneously injected in chickens, resulted in an increment of serum antibody response and cell proliferation response, which are indicators of cellmediated immunity, after an NDV vaccination; thus, these ingredients are advocated as immune potentiators. ${ }^{13}$ The different Chinese herbal treatments used were administrated for three successive days before vaccination. Their NDV vaccination program was two live vaccinations administered at a 14-day interval, with one vaccination administered intranasally and intraocularly; a second vaccination was then injected subcutaneously. They also correlated the effects of herbal treatments on the cell proliferation of the chick embryo fibroblasts (CEFs) in response to an NDV IV vaccine strain in vitro inoculation. ${ }^{13}$ Their results revealed that the herbal treatments enhanced CEF proliferation in vitro in response to the NDV inoculation; this is when NDV inoculation is usually supposed to have a negative effect on CEF proliferation. A similar study evaluated four Chinese herbal polysaccharide effects on a vaccination program that applied an NDV (the Lasota strain) and an infectious bronchitis strain (H120) with live attenuated viruses at 14-day-old chickens by intranasal and intraocular administrations. This was then followed with an ND-IB oil adjuvant vaccine at 28 days by a subcutaneous injection. ${ }^{14}$ The chickens treated with these four different polysaccharide extracts acquired a significant up-regulation in the anti-ND virus HAI antibody titers, above that of the untreated control group, at several time points. These were clearly spotted after the oil adjuvant booster vaccination. In the current study, the aromatic plant essential oil extracts were orally applied, along with the vaccination, in water. This method is surely more practical in the poultry industry when compared to an injectable route for the herbal administration that was applied by previous studies. In addition, the results clearly indicated that a MixOil treatment was successful in boosting the specific and protective humoral immune response generated from a vaccination against NDV.

Most of the studies employing herbal medicine in the animal industries assessed the effects of these supplements on the vaccination-immune response outcome. None of these studies clearly challenged the collected positive results on protection after infection with virulent pathological agents. However, there are a few clinical applications of using herbal medicine in the poultry industry. 15 One of these studies assessed the herbal supplementation effect on production performance, together with the immune status under heat stress. 15 They showed that Ligustrum lucidum and Schisandra chinensis, which are used in Chinese herbal medicines, enhanced egg production, the antibody titers against NDV, lymphocyte proliferation, and the antioxidant condition of the chickens during heat stress. Another study demonstrated an enhancement effect of using the herbal extract in immunesuppressed chickens. 16 The immune suppression in these chickens was induced via reticuloendotheliosis virus (REV) infection. Then, the effect of the herbal treatment, added into the chickens' drinking water, for the whole period of the experiment was evaluated for the vaccination outcome for both the NDV and H5 avian influenza virus vaccines. Their results clearly showed a log difference in the HI antibody titer after treatment with the herbal supplementation in the REV immune-suppressed chickens, and when compared with the untreated immune-suppressed chickens' positive control. Although the main aim of this work has focused on illustrating the effect of MixOil on the immune response after vaccination, the results have clearly indicated a great and positive potential for this product for improving vaccination efficacy after exposure to virulent NDV strains. A decrease in the mortality rate by $50 \%$ after the MixOil immune system-boosting program was clearly reported. Another interesting finding was that, even without exposure to the MixOil immune system-boosting program at the recommended dose before infection at the farm, treatments with a higher dosage of this product at the time of challenge resulted in a similar protective effect. The utmost benefits were achieved when the birds had been placed on the normal recommended manufacture's dose of MixOil before infection at the farm, followed by a high-dose (4X) treatment after the experimental challenge with the virulent virus.
Another further proof of the protection achievement after MixOil treatment is the lower up-regulation in antibody response present in the treated birds after the experimental infection with virulent NDV strains than in the control birds that received no MixOil treatment. The up-regulation in the antibody production in the non-MixOil-exposed groups that had high mortality after the NDV infection points to the inability of the MixOil-untreated group to regulate virus infectivity. In other words, it means that the immune response for the control group was alerted to the extreme after the challenge, resulting in a very high antibody production that was detected in the survived birds 15 days after the experimental challenge. On the other hand, the MixOil-treated groups were able to control the infection process, and this did not lead to a further exaggeration of the antibody response against the NDV challenge. Similarly, the chickens that had a moderate antibody titer detected after the vaccination against NDV, followed by infection with the virulent NDV, only had a slight increase in antibody production after infection. The virulent virus challenge to the nonvaccinated chickens or vaccinated chickens with a low antibody titer achieved from the vaccination resulted in a high antibody response after the challenge. ${ }^{17}$ The spread of the NDV virus in the chickens' tissues (e.g., liver and kidneys) was highest in the vaccinated group that had low antibody production from the vaccination, while the lowest spread of the viruses in tissues was detected in the vaccinated birds that had a moderate antibody titer before the challenge with virulent NDV.17

It is well known that a mass vaccination of non-virulent live virus by spray or via drinking water, which is a method adopted in the poultry industry, produces a variable protection outcome, virus spreading, and antibody immune responses in the vaccinated birds. 18,19 Similarly, the experimental design of this study pointed out that applying, under field conditions, a mass vaccination through the oral administration of a live NDV failed to achieve an adequate protective immunity, even in a healthy flock. This conclusion was further investigated in our laboratory, which proved that, when the birds were orally but individually vaccinated in a controlled environment, a better and more uniform protection outcome was achieved (data not shown).

Many vaccination strategies are adopted in the field, and, according to regional epidemiological situations, a more intensive vaccination program is usually administered. ${ }^{18}$ However, in an endemic area, most vaccination campaigns fail to prevent infection and transmission during outbreaks. An analysis of the causes usually points to the time of vaccination, the vaccine type and formula, the health status of the birds, and the biosecurity 
of the farms. For example, a day 1 vaccination with a live attenuated NDV is usually adopted by most of the farms in an endemic area, such as Jordan. Due to the presence of high maternal antibody titers in vaccinated chicks, this misuse is not usually encouraged by many studies in the literature on vaccination.13,14,20 Although a new in-ova vaccination formula in the form of an antigen antibody complex, or a recombinant vaccination, has been developed, ${ }^{21,22}$ the application of a live attenuated NDV vaccine at one day of age, in its conventional application method, is still the most common malpractice in these endemic areas. Therefore, one cause that might explain the shortage of protection, and the immunity outcome, after the three vaccination doses applied in our current study is the early administration of the vaccine on day 1 ; the average anti-log $\mathrm{HI}$ unit of maternal antibody against the ND virus was around 200 for these birds. This high antibody titer can neutralize the viruses present in the vaccine and thus lead to either a delay or limitation of the immune response generated from the vaccination. ${ }^{23}$ Consequently, one recommendation to be drawn from these results, as well as from other collected data in our laboratory (data not shown), is vaccinations with live attenuated NDV vaccines should be postponed until the chickens are older, by which time the maternal antibody titer will have declined.

This study has investigated, via HAI and ELISA, the immune response outcome after vaccination and MixOil treatment. These two diagnostic techniques are commonly used to assess the humoral immune response in any NDV exposure. The few variations in the antibody response in the figures obtained for both tests could be attributed to the fact that HAI measures both the IgG and IgM agglutination inhibition proprieties, while the ELISA method used only measures IgG. ${ }^{24}$ Isotype switching is expected after infection, and IgG mostly predominates over IgM in a strong vaccination program. 25

The results achieved exceeded expectations and have touched new ground by employing herbal-based products in controlling an NDV infection and decreasing the mortality rate by at least $50 \%$ in the MixOil-treated groups, especially when compared with the untreated group (Table 2). Although the data are not presented in the current study, it is important to mention that preliminary results are accumulating from ongoing field studies in NDV endemic areas such as Jordan, Yemen, and Egypt, confirming similar beneficial effects of using a 4X MixOil formula during NDV outbreaks. Future work can further expand the uses of such herbal products against other viruses facing the poultry industries, and will probably help investigate the mechanisms of action for this product on chicken immunity.

\section{Conclusions}

Finally, the results of this study have demonstrated that the co-administration of aromatic plant essential oil extracts, along with an NDV vaccination, caused a promotion in the efficacy of the vaccination. This was achieved by upregulating the antibody response for NDV, as well as for increasing the protection level achieved from a vaccination against an experimental NDV challenge. Many studies in the literature have presented the boosting effects of diverse herbal extracts on an immune response against viral and bacterial infections and vaccinations in chicken. ${ }^{3,6,7}$ However, according to the authors' knowledge, none of these studies have used in poultry an herbal medicine that has acclaimed a clear and direct benefit in decreasing the mortality rate after a viral infection, or at least specifically after an NDV infection.

\section{References}

1. Solorzano-Santos F, Miranda-Novales MG. Essential oils from aromatic herbs as antimicrobial agents. Curr Opin Biotechnol 2012;23:136-41.

2. Miguel MG. Antioxidant and anti-inflammatory activities of essential oils: a short review. Molecules 2010;15:9252-87.

3. Burt S. Essential oils: their antibacterial properties and potential applications in foods--a review. Int $\mathrm{J}$ Food Microbiol 2004;94:223-53.

4. Greathead H. Plants and plant extracts for improving animal productivity. Proc Nutr Soc 2003;62:279-90.

5. Bozkurt M, Kucukyilmaz K, Catli AU, et al. Performance, egg quality, and immune response of laying hens fed diets supplemented with mannan-oligosaccharide or an essential oil mixture under moderate and hot environmental conditions. Poult Sci 2012;91:1379-86.

6. Bozkurt M, Selek N, Kucukyilmaz K, et al. Effects of dietary supplementation with a herbal extract on the performance of broilers infected with a mixture of Eimeria species. Br Poult Sci 2012;53:325-32.

7. Christaki E, Florou-Paneri P, Giannenas I, et al. Effect of mixture of herbal extracts on broiler chickens infected with Eimeria tenella. Anim Res 2004;53:137-44.

8. Giannenas I, Florou-Paneri P, Papazahariadou M, et al. Effect of dietary supplementation with oregano essential oil on performance of broilers after experimental infection with Eimeria tenella. Arch Tierernahr 2003;57:99-106.

9. Alali WQ, Hofacre CL, Mathis GF, Faltys G.
Effect of essential oil compound on shedding and colonization of Salmonella enterica serovar Heidelberg in broilers. Poult Sci 2013;92:836-41.

10. Ababneh MM, Dalab AE, Alsaad SR, et al. Molecular characterization of a recent Newcastle disease virus outbreak in Jordan. Res Vet Sci 2012;93:1512-4.

11. Sun JL, Hu YL, Wang DY, et al. Immunologic enhancement of compound Chinese herbal medicinal ingredients and their efficacy comparison with compound Chinese herbal medicines. Vaccine 2006;24:2343-8.

12. Guo L, Wang D, Hu Y, et al. Adjuvanticity of compound polysaccharides on chickens against Newcastle disease and avian influenza vaccine. Int $\mathrm{J}$ Biol Macromol 2012;50:512-7.

13. Kong X, Hu Y, Rui R, et al. Effects of Chinese herbal medicinal ingredients on peripheral lymphocyte proliferation and serum antibody titer after vaccination in chicken. Int Immunopharmacol 2004;4:975-82.

14. Qiu Y, Hu YL, Cui BA, et al. Immunopotentiating effects of four Chinese herbal polysaccharides administered at vaccination in chickens. Poult Sci 2007;86:2530-5.

15. Ma D, Shan A, Chen Z, et al. Effect of Ligustrum lucidum and Schisandra chinensis on the egg production, antioxidant status and immunity of laying hens during heat stress. Arch Anim Nutr 2005;59:43947.

16. Liu FX, Sun S, Cui ZZ. Analysis of immunological enhancement of immunosuppressed chickens by Chinese herbal extracts. J Ethnopharmacol 2010;127:2516.

17. Njagi LW, Nyaga PN, Bebora LC, et al. Effect of immunosuppression on newcastle disease virus persistence in ducks with different immune status. ISRN Vet Sci. 2012;2012:253809.

18. van Boven M, Bouma A, Fabri TH, et al. Herd immunity to Newcastle disease virus in poultry by vaccination. Avian Pathol 2008;37:1-5.

19. Senne DA, King DJ, Kapczynski DR. Control of Newcastle disease by vaccination. Dev Biol (Basel) 2004;119:165-70.

20. Fan Y, Lu Y, Wang D, et al. Effect of epimedium polysaccharide-propolis flavone immunopotentiator on immunosuppression induced by cyclophosphamide in chickens. Cell Immunol 2013;281:37-43.

21. Kapczynski DR, Martin A, Haddad EE, King DJ. Protection from clinical disease against three highly virulent strains of Newcastle disease virus after in ovo application of an antibody-antigen complex vaccine in maternal antibody-positive chick- 
ens. Avian Dis 2012;56:555-60.

22. Rauw F, Gardin Y, Palya V, et al. Improved vaccination against Newcastle disease by an in ovo recombinant HVT-ND combined with an adjuvanted live vaccine at day-old. Vaccine 2010;28:823-33.

23. Rauw F, Gardin Y, Palya V, et al. Humoral, cell-mediated and mucosal immunity induced by oculo-nasal vaccination of oneday-old SPF and conventional layer chicks with two different live Newcastle disease vaccines. Vaccine 2009;27:3631-42.

24. Khalifeh MS, Amawi MM, Abu-Basha EA, Yonis IB. Assessment of humoral and cellular-mediated immune response in chickens treated with tilmicosin, florfenicol, or enrofloxacin at the time of Newcastle disease vaccination. Poult Sci 2009;88:211824.

25. Al-Garib S0, Gielkens AL, Gruys DE, et al. Immunoglobulin class distribution of systemic and mucosal antibody responses to Newcastle disease in chickens. Avian Dis 2003;47:32-40. 